\title{
Surface Temperature Measurement in a Fire Environment Using an Infrared Pyrometer
}

\author{
JOE URBAS and WIL L IAM J. PARKER \\ Pacific Fire L aboratory, Inc. \\ 2401 B Talley Way \\ Kelso, WA 98626, USA
}

\begin{abstract}
If a surface temperature measurement were added to a heat release rate test apparatus like the standard ICAL, the net heat flux could be determined. Then the heat of gasification could be calculated for use in the CFD fire models.

This paper describes the measurements of the surface temperature history of specimens in the ICAL using an infrared pyrometer operating in the 8 to 10 micron wavelength band. These temperatures were verified by simultaneous measurements with a bare $0.127 \mathrm{~mm}$ diameter type $\mathrm{K}$ thermocouple pulled tight against the surface of the specimen. As the burning surface receded during the test, the tension, and therefore the surface contact of the thermocouple, was maintained by weights attached to the thermocouple lead wires which passed through two small holes to the back of the specimen.

Because of the strong vibrational bands common to organic polymers, there was very good agreement with the thermocouple for these materials when the spectral emissivity setting of the pyrometer was equal to 1.0.
\end{abstract}

There was essentially no effect of the flames or smoke from the specimens in the ICAL on the temperatures indicated by the pyrometer.

KEYWORDS: fire research, surface temperature, thermocouples, infrared pyrometer, char forming materials, thermoplastic materials, heat flux

\section{INTRODUCTION}

The heat of gasification of a material is one of its most important fire properties. This parameter can be used in Computational Fluid Dynamics (CFD) fire growth models [1] to calculate the mass flow of volatile pyrolysis products from the burning surface. This property can be determined in the Intermediate Scale Calorimeter (ICAL) by dividing the net heat flux by the measured mass loss rate. The net heat flux is equal to the incident flux minus the heat losses. The calculation of the heat losses depends on an accurate measurement of the surface temperature.

The development of an accurate method of measuring the surface temperature is a part of an effort to measure the heat of gasification. The work described in this paper was partially funded by a grant from the Building and Fire Research Laboratory (BFRL) at the National Institute of Standards and Technology (NIST).

In the 1980's an infrared pyrometer was used to measure the surface temperature of wood specimens in the Cone calorimeter [2]. A carefully mounted thermocouple was also attached to the surface so that the spectral emissivity of the specimen could be determined. Then the thermocouple was no longer needed for that material. The best agreement was obtained between the two sensors when the spectral emissivity adjustment 
on the pyrometer was set at 1.0. In order to verify the ability of the pyrometer to measure the temperature of a burning surface it was necessary to maintain a constant tension on the thermocouple so that the junction would remain in contact with the burning surface. It was found that the spectral emissivity of the burning surface was also very close to 1.0.

\section{SURFACE TEMPERATURE MEASUREMENTS ON MATERIALS IN THE VERTICAL ORIENTATION}

In a large in-house project at the Pacific Fire Laboratory the surface temperature histories of ten different materials were measured in the ICAL (described in ASTM E-1623 [3] and ISO/TR 14696 [4]) with a carefully mounted thermocouple and an infrared pyrometer [5]. The $100 \mathrm{~mm}$ x $100 \mathrm{~mm}$ test specimens were in the vertical orientation and were exposed at both 30 and $50 \mathrm{~kW} / \mathrm{m}^{2}$ with at least three replicates. Noncombustible, char forming and thermoplastic materials were all included in the study.

A Heitronics Infrared Pyrometer, Model KT19.81 having a temperature range from 0 to $1000^{\circ} \mathrm{C}$ and a wavelength band of 8-10 microns was used for these tests. The spectral emissivity setting of the pyrometer was maintained at 1.0 during all of the tests. The pyrometer was positioned behind an opening in the ICAL radiant panel. It was aimed just above the thermocouple junction and was viewing a circular target area the size of which depended on the distance between the lens and the target. The distance varied with the incident flux, since the incident flux was determined by the distance between the radiant panel and the specimen. The diameter of the viewed area was $15 \mathrm{~mm}$ and $17 \mathrm{~mm}$ at 50 and $30 \mathrm{~kW} / \mathrm{m}^{2}$, respectively. The infrared pyrometer was calibrated at the factory with a blackbody furnace. Its limit of error is $0.5^{\circ} \mathrm{C}$.

The bare $0.127 \mathrm{~mm}$ diameter type $\mathrm{K}$ thermocouples that were used were available commercially from Omega Engineering, Inc. Their limit of error is $2.2{ }^{\circ} \mathrm{C}$ or $0.75 \%$ of the temperature in ${ }^{\circ} \mathrm{C}$.

Two holes $0.7 \mathrm{~mm}$ in diameter were drilled through the specimen along the horizontal centerline. The centers of the holes were approximately $12.7 \mathrm{~mm}$ apart and equidistant from the center of the specimen. Bare $0.127 \mathrm{~mm}$ diameter, type $\mathrm{K}$ thermocouple leads were then pulled through the holes so that the thermocouple junction was positioned approximately in the middle between the holes on the exposed side of the specimen as seen in Fig. 1. The technique for maintaining good thermocouple contact with the receding surface during burning was developed previously [2] and improved for this work. The leads were protected by small Teflon tubes as they passed through the specimen. This prevented the tar and other pyrolysis products from obstructing the movement of the leads.

The end of each thermocouple lead was secured to a rod behind the specimen. Selected weights were then hung on the thermocouple lead wires approximately in the middle of the leads behind the specimen to provide just enough tension to keep the thermocouple junction and the exposed leads in contact with the surface without pulling them down into the fragile char layer. The range of the weights was between 0.05 and $5 \mathrm{~g}$. Additional replications were sometimes needed to establish the proper amount of tension. The materials tested, their properties, and the results of the measurements are described in [5]. 


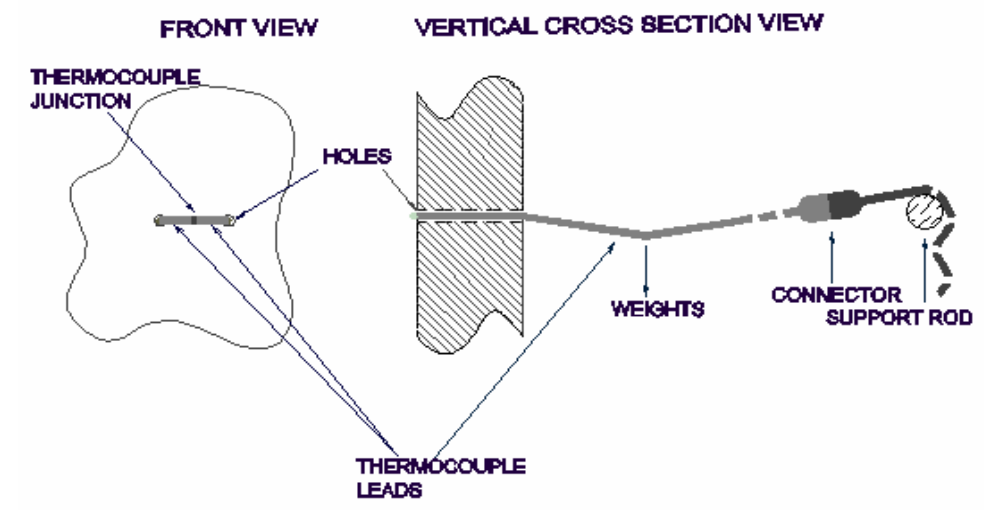

Fig. 1. Experimental setup for thermocouple surface temperature measurements in the vertical orientation.

An example of simultaneous temperature measurements with two sensors on spruce lumber is shown in Fig. 2.

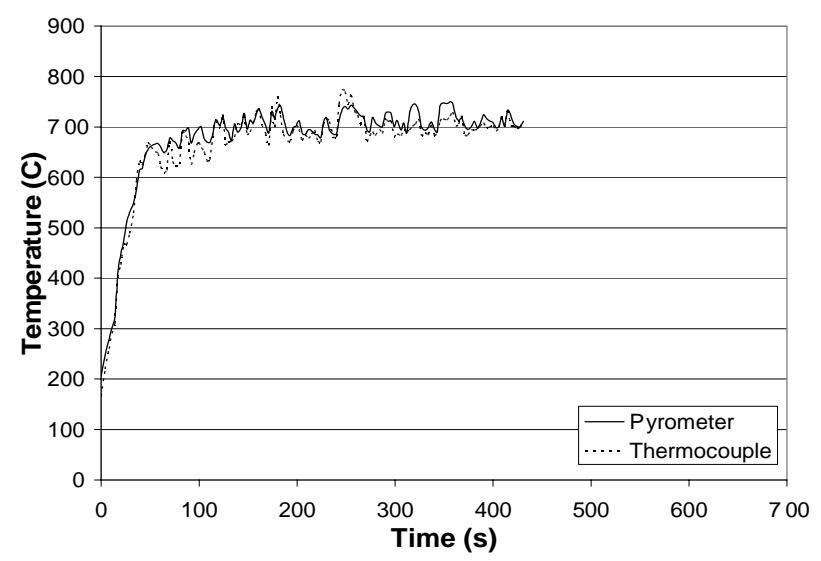

Fig. 2. Spruce lumber at $50 \mathrm{~kW} / \mathrm{m}^{2}$.

Whenever good thermal contact with the surface was obtained for the char forming materials, there was good agreement between the temperatures measured with the thermocouple and the pyrometer. This good agreement was not affected by the flames that were produced after ignition, or by the smoke that was generated before it.

Good thermal contact requires that (1) the thermocouple junction and its lead wires remain in contact with the surface, (2) the diameter be much smaller than the thickness of the thermal boundary layer and (3) if there is a flame present, the diameter be much smaller than the flame quenching distance.

There was good agreement between the temperatures measured with the two different sensors for all of the combustible char forming materials that were tested except when the thermocouple would lose good contact with the surface. Based on this agreement and that of many other previous tests, the infrared pyrometer has been routinely used to measure 
the surface temperature history of all the specimens tested in the ICAL using a spectral emissivity setting of 1.0.

Many attempts were made before such agreement was obtained with a PMMA specimen in the vertical orientation. The pyrometer measures the exposed surface temperature of the thin liquid layer flowing down the surface, but it is extremely difficult to keep the thermocouple on the surface of the liquid. However, the agreement in the test shown in Fig. 3 was sufficient to show that the spectral emissivity of liquid PMMA was close to 1.0. The difference in the two curves prior to ignition was due to a reduction in the tension on the thermocouple in order to let it stay on the surface of the liquid.

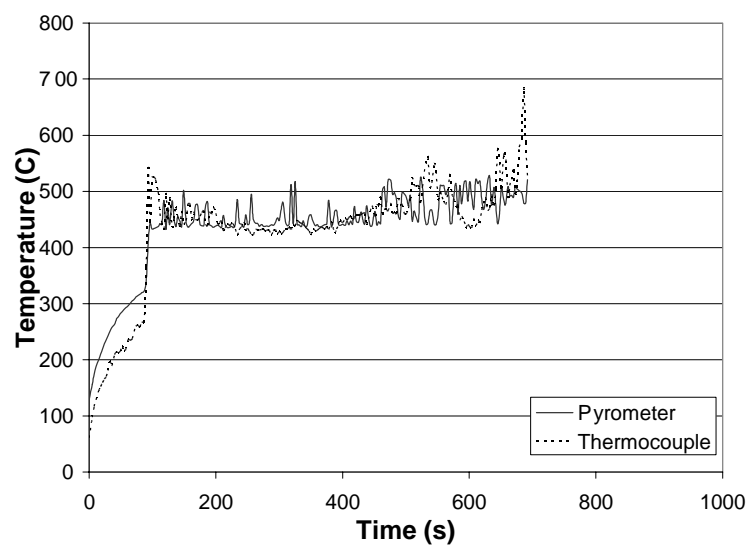

Fig. 3. PMMA at $30 \mathrm{~kW} / \mathrm{m}^{2}$.

\section{Dealing with Reflected Radiation Reaching the Pyrometer}

Since the spectral emissivity is always less than 1.0, there will be some radiation from the radiant panel that is reflected into the pyrometer. However, no noticeable effects of reflection were observed for any of the tested organic materials after ignition. Some materials showed reflection effects before ignition. One inorganic material, the cement board, indicated reflection effects throughout the test. The technique described below was developed to deal with reflection.

The pyrometer calculates the apparent temperature based on the sum of the radiation emitted by the surface and that reflected from it. This is expressed by $q_{p y r}=\varepsilon_{\lambda} q_{o}+q_{\text {ref }}$ where $\mathrm{q}_{\mathrm{o}}$ is the black body flux associated with the actual temperature of the surface and $\mathrm{q}_{\mathrm{ref}}$ is the reflected flux. If it is assumed that the thermocouple provides an accurate surface temperature, the flux seen by the pyrometer can be plotted against the flux associated with the temperature measured by the thermocouple. This will provide a straight line whose slope is equal to the spectral emissivity and an intercept which is equal to the reflected flux as seen in Figures 4 and 5.

This assumes that the spectral emissivity and reflected flux are independent of temperature. While the total emissivity can be a strong function of temperature, the spectral emissivity is usually a constant as long as there are no physical or chemical changes in the surface. If there is a change it will be recognized by a change in the slope of the line. These parameters can also be determined by solving the above equation as a set of simultaneous equations at two different temperatures. 


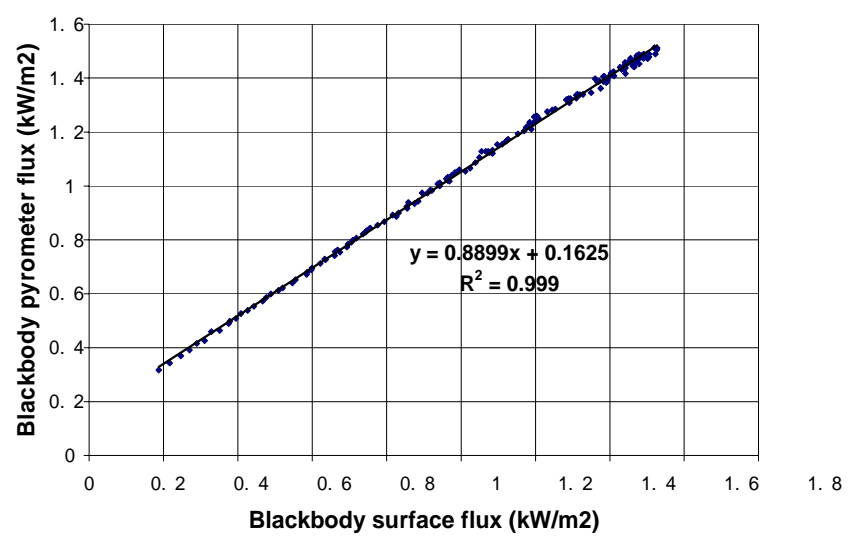

Fig. 4. Plot for determination of the reflected flux and spectral emissivity of cement board exposed at $50 \mathrm{~kW} / \mathrm{m}^{2}$ spectral emissivity $=0.89$, reflected flux $=0.16 \mathrm{~kW} / \mathrm{m}^{2}$.

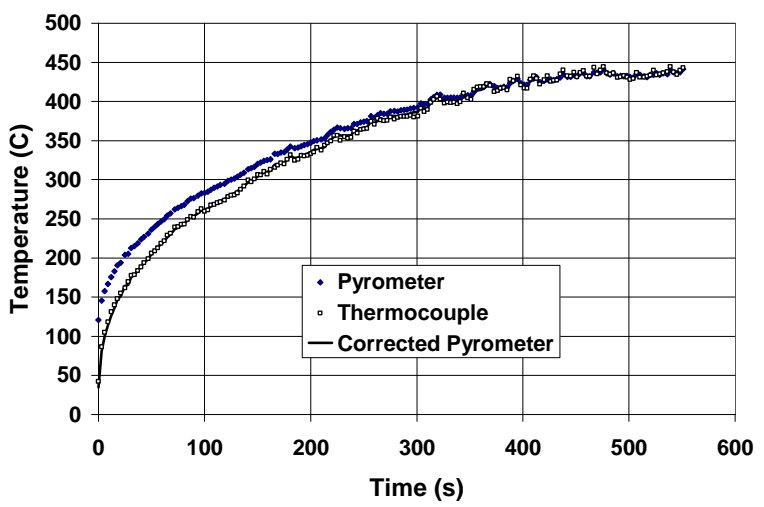

Fig. 5. First $550 \mathrm{~s}$ of the $30 \mathrm{~kW} / \mathrm{m}^{2}$ cement board test including the corrected pyrometer temperature (barely visible due to good agreement with the thermocouple temperature).

The apparent temperature measured by the pyrometer is converted to its associated blackbody flux by Planck's equation. Then it is put into the following formula to get the blackbody flux associated with the surface temperature: $\mathrm{q}_{\mathrm{o}}=\left(\mathrm{q}_{\mathrm{pyr}}-\mathrm{q}_{\mathrm{ref}}\right) / \varepsilon_{\lambda}$.

Finally this is put into an inverted Planck's equation to get the actual surface temperature.

Because of their high spectral emissivity in the wavelength band of the pyrometer, the organic polymers have very low reflected fluxes. The effect of this flux diminishes as the surface temperature rises because the reflected flux is essentially constant while the emitted flux is a strong function of temperature.

\section{SURFACE TEMPERATURE MEASUREMENTS ON MATERIALS IN THE HORIZONTAL ORIENTATION}

These measurements were made on $300 \mathrm{~mm}$ x $300 \mathrm{~mm}$ specimens positioned horizontally in front of the vertical radiant panel of the ICAL, which output was adjusted so that the irradiance over the specimens was uniform within $10 \%$ of the desired value. All the specimens were supported and backed by a $6.35 \mathrm{~mm}$ thick cement board placed on two 
metal beams. The board had two holes drilled $12.7 \mathrm{~mm}$ apart and aligned with the specimen holes for the thermocouple lead passage. The irradiance used for most horizontal tests was $15 \mathrm{~kW} / \mathrm{m}^{2}$; some materials were tested at $25 \mathrm{~kW} / \mathrm{m}^{2}$. Although higher irradiances were attempted first, a very high melting and burning rate of some materials at these irradiances prevented the thermocouple from following the surface at its receding speed. The pyrometer was positioned above the specimen and was viewing the specimen at $26.5^{\circ}$ from the vertical. The diameter of the area on the specimen viewed was $16 \mathrm{~mm}$ at the $26.5^{\circ}$ angle. The pyrometer was protected from the flames of the burning specimens by a calcium silicate insulating board. A metal tube about $150 \mathrm{~mm}$ long was attached to the pyrometer objective. The tube was purged during the tests with nitrogen to prevent accumulation of soot on the pyrometer lens.

The surface temperature history of horizontal specimen in the ICAL was monitored simultaneously by the pyrometer and a thermocouple mounted almost like it was in the standard technique used for the measurements in the vertical orientation. The difference was that the weights $(0.05 \mathrm{~g}-0.85 \mathrm{~g})$ were only used to pull the thermocouple leads downwards. The weights were adjusted by trial and error in attempts to maintain good contact and prevent sinking of the thermocouple into the melting or melted specimen material. The thermocouple leads were fixed at the ends so that the leads formed a Ushaped bend. The weights were pieces of J-shaped wire. They were simply hung on the leads at the lowest point of the bend.

It was found that the pyrometer reading was not affected by viewing the surface at an angle of $26.5^{\circ}$ to the normal. This allowed the pyrometer to be located in a safe area away from the flame. The use of a slanted nitrogen purged pipe allowed the temperature measurements to be made well inside of the specimen boundary and still make it through a limited path length in the flames and smoke.

The specimens were ignited with a small flame.

The materials and their properties are given in Table 1.

Table 1. Materials tested.

\begin{tabular}{|c|l|c|r|}
\hline No. & Material & Density $\left.\mathbf{( k g} / \mathbf{m}^{\mathbf{3}}\right)$ & Thickness (mm) \\
\hline 1. & Hardboard & 806 & 12.7 \\
\hline 2. & PU Flexible Foam & 25.4 & 49 \\
\hline 3. & Clear PMMA & 1175 & 12.5 \\
\hline
\end{tabular}

\section{Results and Discussion of Hardboard Experiments}

While making surface temperature measurements on hardboard in the horizontal orientation during the present project, it was found that the thermocouple mounting procedure described above needed to be expanded at least for hard materials.

For the tests on hardboard, applying a very high tension could not bring the thermocouple into good thermal contact with the surface as seen in Fig. 6. It was concluded that it was due to the inability of the lead wires to complete a right angle bend within the hole. If the bend has to be completed before going into the hole, there will be some small separation between the lead and the surface. If the wire is pulled tight between the two holes the junction will be pulled away from the surface by a minute amount. In the case of a soft or fragile material the bending could take place inside the material around the hole. Then the 
lead wire could remain in thermal contact with the surface until it was gradually pulled into the fragile material around the hole and then into it preventing good thermal contact.

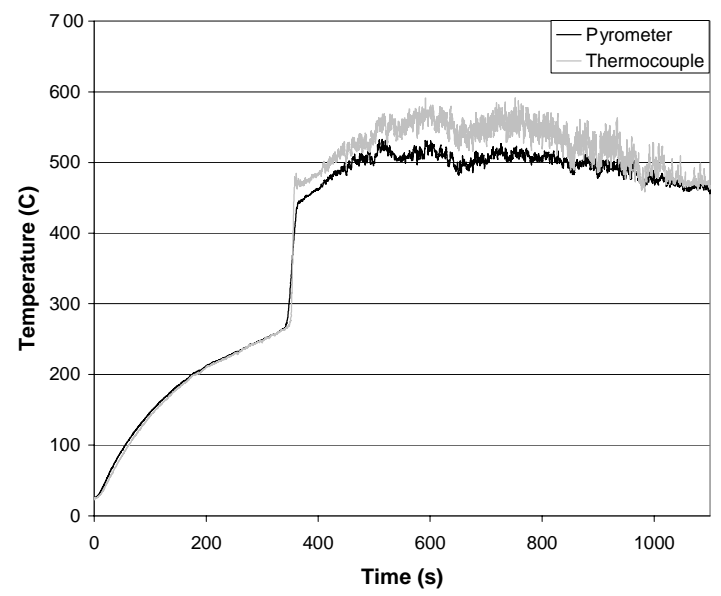

Fig. 6. Hardboard at $15 \mathrm{~kW} / \mathrm{m}^{2}$ in the horizontal orientation, without pull down wires.

The solution was to pull each lead wire flat against the surface at a point on each side of the junction. This was accomplished by drilling two $0.7 \mathrm{~mm}$ diameter holes about $2 \mathrm{~mm}$ from each side of the junction. A $0.0508 \mathrm{~mm}$ wire was threaded through each hole making a loop over the thermocouple lead and then pulled back through the hole. Then a weigh was attached. This experimental setup is shown in Fig. 7.

The result was good agreement between the two sensors up to 700 seconds as seen in Fig. 8. The use of these pull down wires should be considered whenever the surface temperature of burning specimens is being measured.

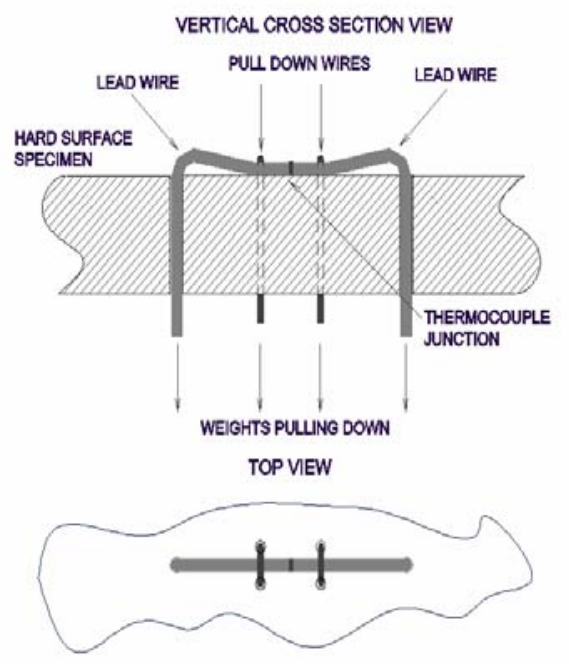

Fig. 7. Experimental setup for the thermocouple measurements of surface temperature on hard surface. 


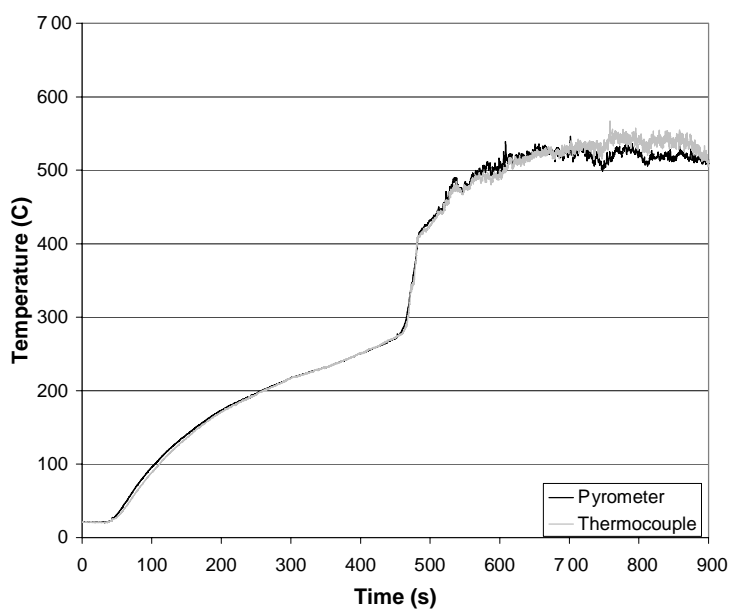

Fig. 8. Hardboard at $15 \mathrm{~kW} / \mathrm{m}^{2}$ in the horizontal orientation, with pull down wires.

\section{Analysis of PMMA Data Curve}

Figure 9 is a good illustration of what can be accomplished when a thermocouple and a pyrometer are used together to measure the surface temperature of a horizontal specimen of clear PMMA in the ICAL. Prior to ignition the smooth curve provided by the thermocouple indicated that it was being held in good thermal contact with the surface and thus provided an accurate value of the surface temperature. The spectral emissivity setting used for the infrared pyrometer was always equal to 1.0 in these tests. The fact that the pyrometer curve is almost indistinguishable from that of the thermocouple means that the spectral emissivity of clear PMMA is very close to 1.0 in the wavelength band of the pyrometer which is 8 to 10 microns.

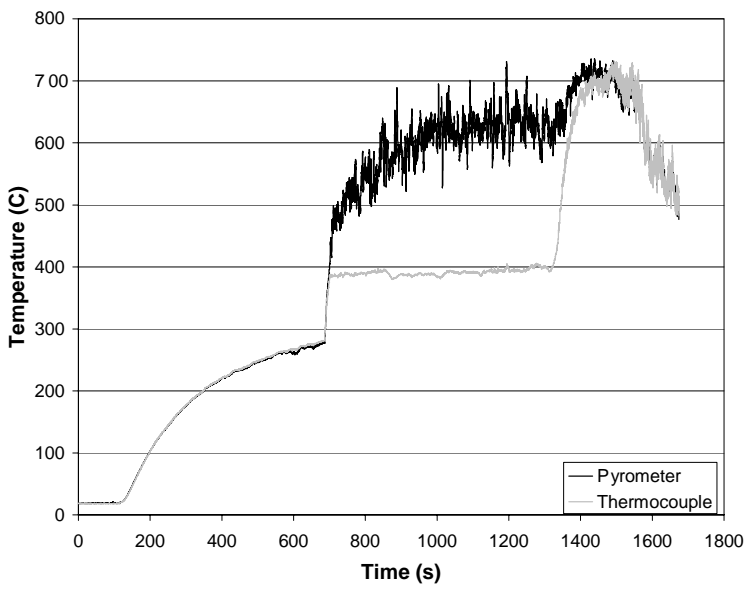

Fig. 9. Clear PMMA exposed at $15 \mathrm{~kW} / \mathrm{m}^{2}$.

When the surface temperature reaches about $270{ }^{\circ} \mathrm{C}$, the PMMA molecules begin to depolymerize to produce a MMA vapor. When a flammable mixture of MMA and air is reached above the specimen, a flaming ignition will occur in the presence of a pilot 
igniter. The sudden increase in net heat flux causes a rapid increase in the surface temperature. When this reaches about $390{ }^{\circ} \mathrm{C}$, the pyrolysis becomes more vigorous due to random scissions along with the unzipping.

The pyrolysis products now include liquids as well as gases. A liquid layer begins to form on top of the PMMA substrate. It is interesting to note that in the thermogravimetric studies conducted by Zhang and Blum [6] on specimens of isotactic PMMA adsorbed on silica, that degradation peaks were observed at $150{ }^{\circ} \mathrm{C}, 260{ }^{\circ} \mathrm{C}$ and $390{ }^{\circ} \mathrm{C}$. Thus the peak pyrolysis rates suggested by the surface temperature measurements in this project appear to correlate with the peak mass loss rates at the two higher temperatures in the TGA studies.

Tension must be maintained on the thermocouple lead wires in order to keep the junction tight against the surface, even when the wire is lengthening due to thermal expansion. The liquid layer cannot support the thermocouple junction under these conditions. Consequently, the thermocouple is pulled tight against the PMMA surface at the bottom of the liquid layer and continues to read a temperature around $390{ }^{\circ} \mathrm{C}$ throughout most of the burning period. The relatively smooth line suggests that the thermal contact is good so that the temperature being measured is actually that of the surface of the solid PMMA.

The pyrometer sees the radiation from the upper surface of the liquid layer. Since it is an organic material its spectral emissivity is probably close to unity between 8 and 10 microns. This has yet to be proven. What appears to be a noise band is probably due to the fluctuation of the surface temperature due to bubbling. When the solid PMMA is completely gone, the energy that had been going into pyrolysis is raising the temperature of the liquid layer. Finally as the thickness of this liquid layer and the temperature drop across it go to zero, the temperatures measured with the thermocouple and the pyrometer come back into good agreement. Good agreement occurring during the liquid only burning period suggests that the spectral emissivity of the liquid is also close to unity. If that is true, then the pyrometer is following the temperature of the surface throughout the test, regardless of whether it is solid or liquid. It should be noted that the pyrolysis of the solid PMMA is taking place in an inert atmosphere. The liquid layer serves as a "getter" to exclude any atmospheric oxygen.

\section{Analysis of the Soft Polyurethane Foam Data Curve}

There are some similarities in the data curves of PU foam in Fig. 10 and the PMMA in Fig. 9. Ignoring the large sharp spike in the thermocouple curve as a noise spike of unknown origin, the first of the two major peaks in the pyrometer curve takes place during the melting phase. During this time the thermocouple is loosely attached to the surface of the solid PU which is separated from the exposed surface by a growing layer of melted PU. The pyrometer is recording the temperature of the liquid surface. Heat losses start to occur as the liquid approaches the bottom of the specimen holder. This slows down the rate of melting and thus diminishes the temperature difference between the two sensors. After the solid is completely consumed, the thermocouple is loosely attached to the liquid surface. From then on the thermocouple and the pyrometer should be reading the same temperature if the liquid is black in the 8 to 10 micron band. The deviations between the readings of the pyrometer and the thermocouple during this period are assumed to be due to the difficulty in maintaining good thermocouple contact. 


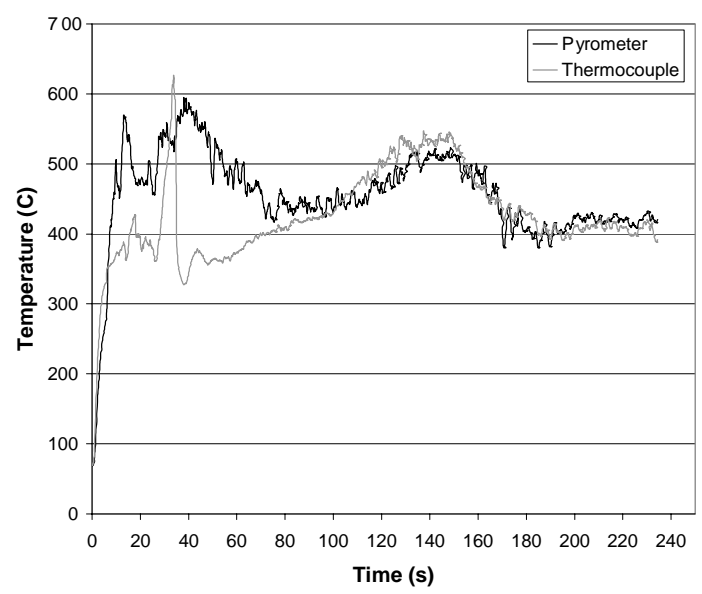

Fig. 10. Soft PU foam at $25 \mathrm{~kW} / \mathrm{m}^{2}$ in the horizontal orientation.

\section{GENERALIZATION TO ALL ORGANIC POLYMERS}

All of the combustible materials, that the authors of this paper have made simultaneous surface temperature measurements on with a thermocouple and an infrared pyrometer operating in the 8 to 10 micron wavelength band, have had a spectral emissivity approaching 1.0 in that spectral region. Measurements have not been made over enough different materials to make that claim for all combustible materials. However, we are finding information in the literature that might form a basis for that claim.

Fig. 11 is taken from "The Textbook of Polymer Science" by Fred Billmeyer [7]. It displays the rich variety of vibrational bands in the thermal infrared. Note that the spectral band associated with the Carbon-Carbon single bond, which is found in virtually every organic polymer, covers almost the entire 8-10 micron band of this infrared pyrometer. It is completely covered by the Carbon-Oxygen and Carbon-Nitrogen single bonds. Therefore it is likely that all organic polymers will exhibit high spectral emissivities in this wavelength band. If this is proven to be true it will greatly simplify the surface temperature measurements. 


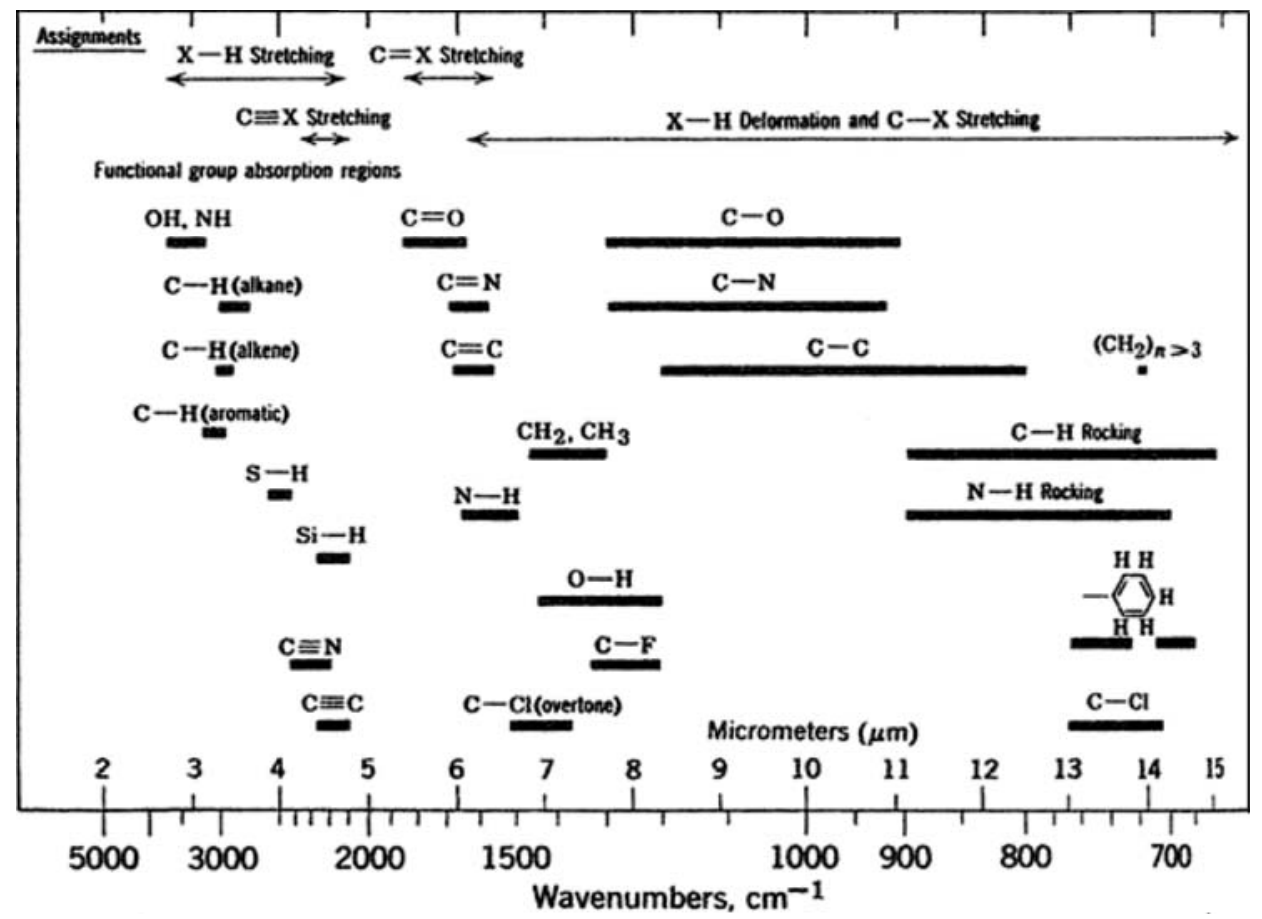

Fig. 11. Vibrational bands in the thermal infrared.

In the same book it states that "One of the greatest experimental difficulties in work with substances that absorb heavily in the infrared, including many polymers, is obtaining sufficiently thin samples." Otherwise there will be an overlapping of the absorption bands which increases with penetration. This could lead to the high spectral emissivities quoted in the next reference.

Jackson [8] states that "The thermal infrared spectral region of 8 to 13 microns is typically used for remote thermal sensing. This spectral range contains the maximum thermal emission for temperatures in the range found at the earth's surface and is less subject to absorption by atmospheric gases. Plant leaves have spectral emissivity values that typically range between 0.97 and 0.98 . Assuming a spectral emissivity of 1.0 for plants will usually result in less than $1.0^{\circ} \mathrm{C}$ error." This could amount to around $3{ }^{\circ} \mathrm{C}$ for a burning surface at $700{ }^{\circ} \mathrm{C}$.

It is relatively easy to check whether the spectral emissivity is close to unity for a particular material by making simultaneous surface temperature measurements with the infrared pyrometer that was used on this project and a properly mounted thermocouple, following the procedure employed on this project. The term, spectral emissivity, in this paper refers to its value in the wavelength band of the pyrometer.

For this spectral emissivity check, the range would be kept below the melting point and the threshold temperature of active pyrolysis. Hence there will be no movement of the front surface. Therefore, the thermocouple leads coming through the holes to the rear surface would only need to be secured in place. If there is a change in the spectral emissivity for a particular organic material with increasing temperature, it will be due to 
an increase in the number of populated energy levels, which would result in a higher spectral emissivity.

\section{CONCLUSIONS}

1. An infrared pyrometer operating in the 8 to 10 micron wavelength band with a fixed spectral emissivity setting of 1.0 can measure the surface temperature histories of organic materials with acceptable accuracy in the vertical and horizontal orientations. This includes the exposed surfaces of the downward flow of melting thermoplastics.

2. The surface temperature measurement of a burning specimen in the ICAL is not affected by its flame or its smoke.

3. It is likely that nearly all organic polymers have high spectral emissivities in the 8 to 10 micron wavelength band.

4. Satisfactory surface temperature measurements can be made with bare $0.127 \mathrm{~mm}$ type $\mathrm{K}$ thermocouples if the junction and the adjacent leads are maintained in good thermal contact with the surface.

\section{REFERENCES}

[1] McGrattan, K.B., "Fire Dynamics Simulator (Version 4) - Technical Reference Guide,” National Institute of Standards and Technology, NIST Special Publication 1018, Gaithersburg, MD., July 2004, 94 p.

[2] Urbas, J., Parker, W.J., "Surface Temperature Measurements on Burning Wood Specimens in the Cone Calorimeter and the Effect of Grain Orientation,” Fire and Materials, 17: 205-208 (1993).

[3] ASTM, E-1623, Standard Test Method for Determination of Fire and Thermal Parameters of Materials, Products, and Systems Using an Intermediate Scale Calorimeter (ICAL).

[4] ISO/TR 14696, Reaction to Fire Tests, Determination of Fire Parameters of Materials, Products, and Assemblies Using an Intermediate Scale Calorimeter (ICAL), Geneva, 1999.

[5] Urbas, J., Parker, W.J., and Luebbers, G.E., "Surface Temperature Measurements on Burning Materials Using an Infrared Pyrometer: Accounting for Spectral Emissivity and Reflection of External Radiation,” Fire and Materials, 28: 33-53 (2004).

[6] Zhang, B. and Blum, F.D., "Thermogravimetric Studies of PMMA on Silica," Polymer Reprints, 43 (17): 484 (2002).

[7] Billmeyer, F., Textbook of Polymer Science, $3^{\text {rd }}$ Edition, John Willey and Sons Inc., NY (1984).

[8] Jackson, R.D., "Canopy temperature and crop water stress," Advances in Irrigation, Volume 1, Hillel, D.I., (ed.), Academic Press, 1982, p. 43/85. 